\title{
Microrganismos multifuncionais: utilização na agricultura
}

\author{
Multifunctional microorganisms: use in agriculture \\ Microorganismos multifuncionales: uso em agricultura
}

Recebido: 05/02/2021 | Revisado: 15/02/2021 | Aceito: 18/02/2021 | Publicado: 27/02/2021

\author{
Cássia Cristina Rezende \\ ORCID: https://orcid.org/0000-0001-8463-1907 \\ Universidade Federal de Goiás, Brasil \\ E-mail: cassiacristinarezende@ hotmail.com \\ Mariana Aguiar Silva \\ ORCID: https://orcid.org/0000-0003-0297-5576 \\ Universidade Federal de Goiás, Brasil \\ E-mail: marianaaguiar23@hotmail.com \\ Laylla Luanna de Mello Frasca \\ ORCID: https://orcid.org/0000-0002-3572-1145 \\ Universidade Federal de Goiás, Brasil \\ E-mail: layllafrasca@gmail.com \\ Denner Robert Faria \\ ORCID: https://orcid.org/0000-0002-7779-9987 \\ Universidade Federal de Goiás, Brasil \\ E-mail: dennerobertpgtu@gmail.com \\ Marta Cristina Corsi de Filippi \\ ORCID: https://orcid.org/0000-0003-1676-8164 \\ Embrapa Arroz e Feijão, Brasil \\ E-mail: cristina.filippi@embrapa.br \\ Anna Cristina Lanna \\ ORCID: https://orcid.org/0000-0001-8018-9349 \\ Embrapa Arroz e Feijão, Brasil \\ E-mail: anna.lanna@embrapa.br \\ Adriano Stephan Nascente \\ ORCID: https://orcid.org/0000-0002-6014-3797 \\ Embrapa Arroz e Feijão, Brasil \\ E-mail: adriano.nascente@embrapa.br
}

\begin{abstract}
Resumo
Microrganismos multifuncionais são microrganismos benéficos que apresentam mecanismos diretos e indiretos de promoção de crescimento vegetal e apresentam um papel importante como uma tecnologia alternativa rumo á escala para uma agricultura sustentável. O aumento da demanda pela produção agrícola com uma redução significativa do uso de fertilizantes e pesticidas sintéticos é um grande desafio na atualidade. O estudo desses microrganismos vem merecendo destaque nos últimos anos em razão da grande demanda por tecnologias sustentáveis, podendo reduzir os custos de produção, aumentando a produtividade e a rentabilidade do agronegócio. A aplicação de microrganismos multifuncionais através de mecanismos diretos e indiretos tem mostrado que é possível tornar as práticas de manejo das culturas ambientalmente mais sustentáveis. Os mecanismos dos microrganismos multifuncionais incluem a regulação do equilíbrio hormonal, solubilização de nutrientes na solução do solo e indução de resistência contra patógenos. O objetivo do trabalho foi trazer informações referentes aos microrganismos multifuncionais priorizando os aspectos gerais, as características dos microrganismos (rizobactérias e fungos) promotores do crescimento vegetal e seus principais mecanismos de ação. Em resumo, conclui-se que os microrganismos multifuncionais possuem diversas características favoráveis para serem utilizados como promotores de crescimento das plantas, na agricultura sustentável.
\end{abstract}

Palavras-chave: Produção agrícola; Rizobactérias promotoras de crescimento vegetal; Fungo; Sustentabilidade.

\begin{abstract}
Multifunctional microorganisms are beneficial microorganisms that have direct and indirect mechanisms for promoting plant growth and play an important role as an alternative technology towards scale for sustainable agriculture. The increase in demand for agricultural production with a significant reduction in the use of synthetic fertilizers and pesticides is a major challenge today. The study of these microorganisms has been highlighted in recent years due to the great demand for sustainable technologies, which may reduce production costs, increasing the productivity and profitability of agribusiness. The application of multifunctional microorganisms through direct and indirect mechanisms has shown that it is possible to make crop management practices more environmentally sustainable. The mechanisms of multifunctional microorganisms include the regulation of hormonal balance,
\end{abstract}


solubilization of nutrients in the soil solution and induction of resistance against pathogens. The objective of the work was to bring information regarding multifunctional microorganisms prioritizing the general aspects, the characteristics of the microorganisms (rhizobacteria and fungi) that promote plant growth and their main mechanisms of action. In summary, it is concluded that multifunctional microorganisms have several favorable characteristics to be used as promoters of plant growth in sustainable agriculture.

Keywords: Agricultural production; Plant growth promoting rhizobacteria; Fungi; Sustainability.

\section{Resumen}

Los microorganismos multifuncionales son microorganismos beneficiosos que tienen mecanismos directos e indirectos para promover el crecimiento de las plantas y juegan un papel importante como tecnología alternativa a escala para la agricultura sostenible. El aumento de la demanda de producción agrícola con una reducción significativa en el uso de fertilizantes y pesticidas sintéticos es un desafío importante en la actualidad. El estudio de estos microorganismos se ha destacado en los últimos años debido a la gran demanda de tecnologías sostenibles, que pueden reducir los costos de producción, aumentando la productividad y rentabilidad de la agroindustria. La aplicación de microorganismos multifuncionales a través de mecanismos directos e indirectos ha demostrado que es posible hacer que las prácticas de manejo de cultivos sean más sostenibles ambientalmente. Los mecanismos de los microorganismos multifuncionales incluyen la regulación del equilibrio hormonal, la solubilización de nutrientes en la solución del suelo y la inducción de resistencia contra patógenos. El objetivo del trabajo fue traer información sobre los microorganismos multifuncionales priorizando los aspectos generales, las características de los microorganismos (rizobacterias y hongos) que promueven el crecimiento de las plantas y sus principales mecanismos de acción. En resumen, se concluye que los microorganismos multifuncionales tienen varias características favorables para ser utilizados como promotores del crecimiento vegetal en la agricultura sostenible.

Palabras clave: Producción de agricultura; Rizobacterias promotoras del crecimiento vegetal; Hongos; Sustentabilidad.

\section{Introdução}

A utilização massiva dos agrotóxicos se iniciou na década de 60, na chamada Revolução Verde - um movimento pela modernização da agricultura, utilizando máquinas, agrotóxicos e sementes geneticamente modificadas com o intuito de aumentar a produtividade. A partir desse momento, grande parte dos agricultores brasileiros passaram a utilizar esses produtos, inclusive pequenos agricultores (Carvalho \& Chagas, 2019).

Porém, essa ação desencadeou uma degradação das terras agrícolas causada pela ação do homem no manejo do solo e das culturas, que afeta de maneira irreversível extensas áreas cultivadas e contribui para o decréscimo do rendimento das culturas (Rai et al., 2020). Este tipo de produção, considerada não sustentável, acarreta também problemas ambientais como poluição dos recursos hídricos, do solo e do ar; além de riscos econômicos como o aumento dos custos de produção (Pietro Souza et al., 2020). Assim, devido precauções com a saúde e meio ambiente intensificaram o interesse para o desenvolvimento de tecnologias alternativas, garantindo rendimentos competitivos e proteção das culturas, em uma abordagem sustentável levando a um equilíbrio ecológico à longo prazo (Singh et al., 2017).

Práticas de manejo de culturas estabelecidas a algumas décadas atrás, como rotação de culturas, desenvolvimento de cultivares com características agronômicas e fisiológicas de interesse e o uso de plantas de cobertura quando utilizadas isoladamente, não estão sendo suficientemente adotadas para mitigar os impactos negativos da degradação das áreas cultivadas sem sustentabilidade (Abhilash et al., 2016). Para Lopes et al. (2020), essas práticas devem ser complementadas com alternativas racionais e seguras, como o uso de microrganismos multifuncionais, principalmente, rizobactérias promotoras de crescimento vegetal e fungos de solo como o Trichoderma sp.

As rizobactérias promotoras de crescimento vegetal (RPCV) são definidas como bactérias que habitam a rizosfera de plantas realizando funções que promovem o crescimento vegetal e são beneficiadas pelos exsudados das raízes (Ahemad \& Kibret, 2014). De acordo com Singh et al. (2017), essas rizobactérias exercem efeitos benéficos sobre as plantas por diferentes mecanismos de ação, sendo diretos (fixação biológica de nitrogênio, solubilização do fosfato, produção de fitormônios) ou indiretos (produção de sideróforos e biofilme). Vários gêneros de bactérias (Acetobacter, Azospirillum, Azotobacter, Bacillus, Burkholderia, Klebsiella, Pseudomonas e Serratia) foram relatados como capazes de promover o crescimento das plantas, 
incrementos na produtividade de grãos, aumento na emergência de sementes, biomassa vegetal e rendimento da colheita; e resistência ás doenças (Kang et al., 2019).

Algumas espécies de fungos também apresentam potencial em promover crescimento em plantas (Cadore et al., 2018). O gênero Trichoderma sp., considerado como um dos mais explorados, é composto de fungos de vida livre e apresenta importância devido ao seu grande potencial de aplicação do ponto de vista agrícola, industrial e biotecnológico (Chagas et al., 2017). Diante deste desafio de produzir de forma mais sustentável, a produção de produtos biológicos para controle de pragas e doenças agrícolas cresceu mais de 70\% no último ano no Brasil, movimentando $\mathrm{R} \$ 464,5$ milhões. O resultado brasileiro é considerado o mais expressivo da história do setor e supera o percentual apresentado pelo mercado internacional (Brito, 2020).

Porém, devido as inconsistências nos trabalhos de pesquisa com esses microrganismos sob condições de campo, em todo o mundo, poucas são as espécies de RPCVs que são efetivamente transformadas em produtos comerciais para utilização em larga escala (Steffen et al., 2018). Estudos sobre o ambiente, a cultura, as condições do solo e as interações com os microrganismos nativos do solo são fatores que necessitam de investigação aprofundada e que podem contribuir para o avanço do conhecimento e a aplicabilidade nos sistemas agrícolas diversos (Kang et al., 2019).

Dessa forma, essa revisão objetiva trazer informações referentes aos microrganismos multifuncionais priorizando os aspectos gerais, as características dos microrganismos (rizobactérias e fungos) promotores do crescimento vegetal e seus principais mecanismos de ação.

\section{Desenvolvimento}

Trata-se de um estudo de caráter teórico, através de uma pesquisa bibliográfica de caráter exploratório e abordagem qualitativa (Pereira et al., 2018), que envolveu, fundamentalmente, a análise de informações e características sobre os microrganismos multifuncionais e seus mecanismos de ação.

\section{Aspectos Gerais dos Microrganismos Multifuncionais}

Diversos microrganismos quando associados à planta podem promover o seu crescimento e uma série de outros benefícios no seu desenvolvimento e são referidos na literatura como microrganismos promotores do crescimento vegetal (Abhilash et al., 2016). Esses microrganismos podem ser considerados insumos tecnológicos no contexto da economia verde, pois reduzem o uso de insumos sintéticos na produção agrícola (Chagas et al., 2017), representando assim alternativa estratégica para a intensificação sustentável de sistemas agrícolas (Steffen et al., 2018).

A rizosfera, camada do solo mais próxima das raízes, é o lugar onde ocorre o maior número de interações entre plantas e microrganismos (Bhattacharyya \& Jha, 2012). Essa região compõe um habitat ideal para os microrganismos multifuncionais, pois nela encontram-se uma gama de compostos liberados pelas plantas (exsudatos radiculares), os quais são utilizados no metabolismo e crescimento microbiano (Rout, 2014). A interação dos microrganismos com as plantas na rizosfera pode ocorrer de diferentes formas: microrganismos de vida livre localizados na matriz do solo rizosférico, microrganismos associados à superfície da raiz da planta ou rizoplano e microrganismos que se encontram dentro dos tecidos da raiz, nos espaços intercelulares ou intracelulares, sendo conhecidos como endofíticos (Santoyo et al., 2016).

Os mecanismos pelos quais os microrganismos multifuncionais promovem o crescimento das plantas podem ser diretos ou indiretos (Vejan et al., 2016). Diretamente, aprimorando a absorção e acúmulo de nutrientes essenciais às plantas (Schlaeppi \& Bulgarelli, 2015), fixação biológica de nitrogênio, solubilização de fosfato, secreção de sideróforos (Bashan et al., 2013) e produção de fitormônios (González et al., 2011). Indiretamente, destacam-se o biocontrole contra pragas e fitopatógenos (Liotti et al., 2018) e a indução de resistência das plantas aos estresses bióticos e abióticos como metais pesados, deficiência hídrica e salinidade (Pietro Souza et al., 2020). Silva et al. (2020) verificaram incremento de $25 \%$ na produção de 
biomassa de raiz de plantas de soja microbiolizadas com isolados de microrganismos multifuncionais (RPCVs e o fungo Trichoderma asperellum) quando comparados ao controle (sem microbiolização).

A promoção do crescimento radicular também é um dos efeitos benéficos dos microrganismos multifuncionais, pois o estabelecimento rápido das raízes laterais e adventícias é uma característica vantajosa para as plantas, aumentando a habilidade destas de se fixar ao solo e obter água e nutrientes do ambiente (Moreira \& Araújo, 2013). Sousa et al. (2019), trabalhando com arroz irrigado por inundação, verificaram que sementes da cultivar BRS Catiana tratadas com os isolados BRM32110 e BRM32112 apresentaram aumento médio de 25,7\% no comprimento radicular, em comparação com o tratamento controle, enquanto para a cultivar BRS A702 CL, todos os microrganismos proporcionaram incrementos com um valor médio de $31 \%$ no comprimento radicular das plântulas de arroz.

A Figura 1 mostra os efeitos benéficos das bactérias promotoras de crescimento vegetal simbiônticas e nãosimbiônticas, apresentando os mecanismos como indução de resistência sistêmica em nematoides e patógenos, síntese de hormônios vegetais (auxina, etileno e giberelina), aumento de tolerância vegetal e estresses abióticos, disponibilização de nutrientes e manutenção das características desejáveis do solo.

Figura 1. Efeitos benéficos das bactérias promotoras de crescimento vegetal (BPCV), simbiônticas ou não-simbiônticas, no desenvolvimento da cana-de-açúcar.

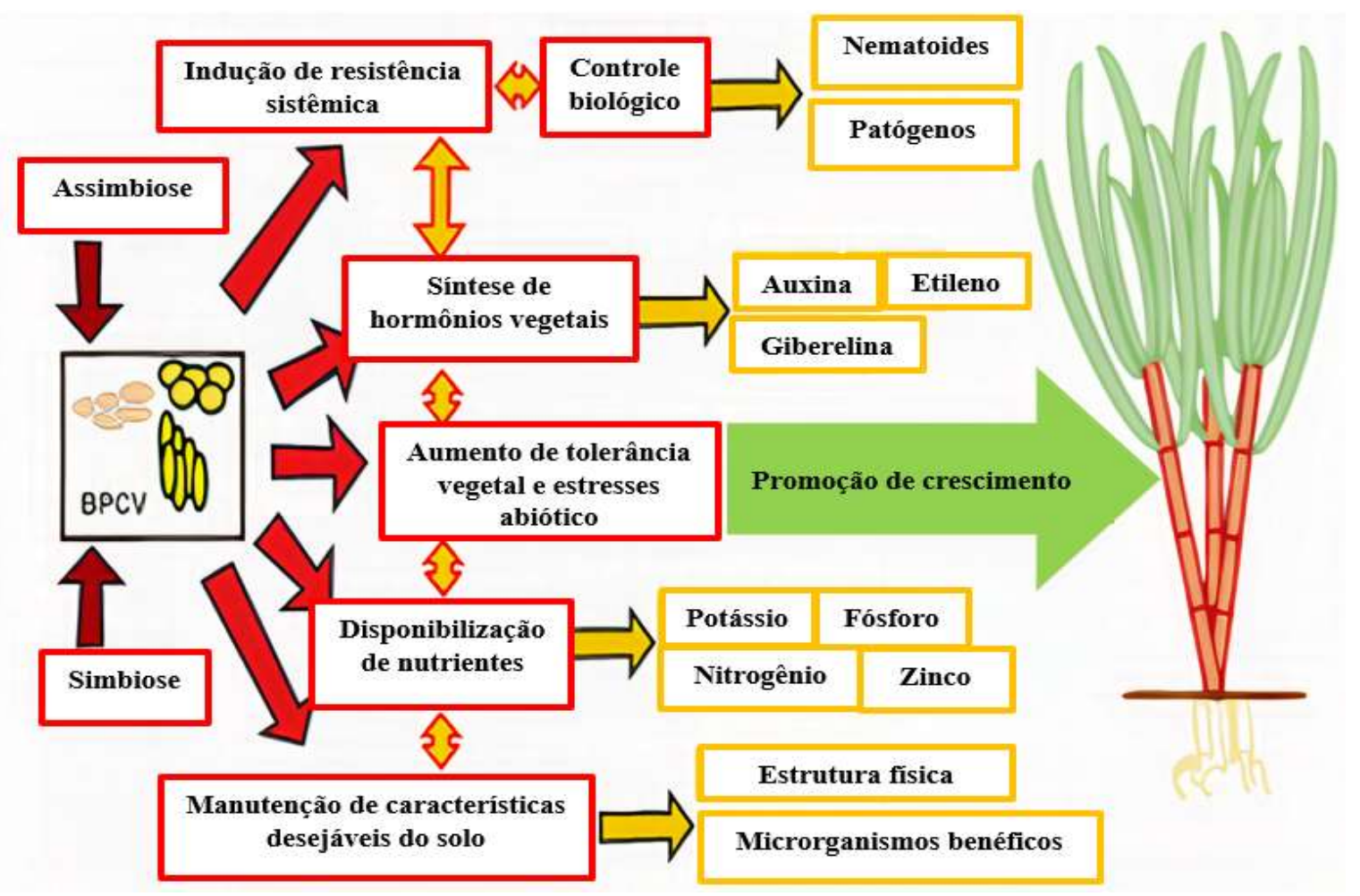

Fonte: Márcia Eugênia Amaral Carvalho (2015) (Adaptado de Maheshwari, 2011).

\section{Rizobactérias Promotoras de Crescimento Vegetal (RPCVs)}

Dentre os microrganismos presentes na rizosfera existe uma população variada de bactérias, denominadas rizobactérias. Essas são conhecidas como RPCVs quando apresentam efeitos benéficos sobre as culturas agrícolas (Montaldo, 2016). A promoção do crescimento vegetal pelas RPCVs está ligada a fatores como maior taxa germinativa das sementes, maior concentração de nutrientes nas plantas, aumento no acúmulo de fitomassa e altura de plantas, maior produção de grãos, dentre outros componentes da produtividade (Ratz, 2017). 
As RPCVs, dependendo da proximidade, do grau de intimidade que estabelecem com as raízes e com as células do hospedeiro, podem ser classificadas em dois grupos: eRPCV ("rizobactérias promotoras de crescimento vegetal extracelulares") e iRPCV (“rizobactérias promotoras de crescimento vegetal intracelulares”) (Vejan et al., 2016) (Figura 2). As eRPCV são aquelas presentes na rizosfera e rizoplano ou nos espaços intercelulares das células do córtex radicular tais como Azospirillum sp., Bacillus sp., Burkholderia sp., Pseudomonas sp.e Serratia sp. Exemplos de iPGPR são Bradyrhizobium e Rhizobium, normalmente encontradas em estruturas nodulares especializadas da raiz (Martínez Viveros et al., 2010).

Figura 2. iRPCV X eRPCV associadas com as raízes de plantas.

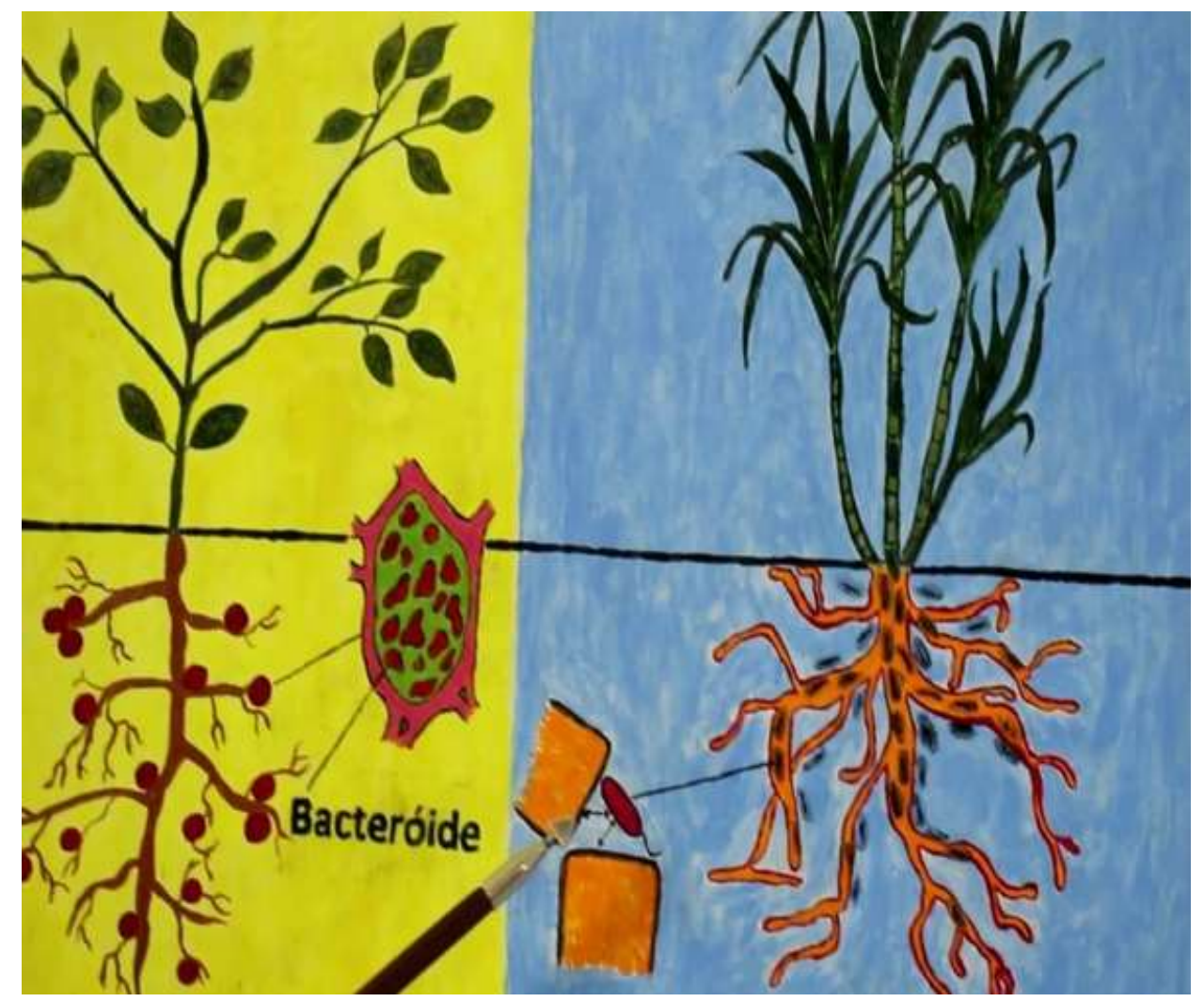

Fonte: Armando Silva (2016).

A eficiência das RPCVs pode ser incrementada pela técnica de co-inoculação, que consiste na combinação de isolados potenciais de diferentes espécies para somar os efeitos plurifacetados durante a interação microrganismos-planta (Dalolio et al., 2018). Resultados positivos para o crescimento do milho derivados do uso combinado de RPCVs foram obtidos por Akhtar et al. (2014) ao verificarem aumento da altura, diâmetro de colmo, massa seca de parte aérea e dos teores de fósforo e nitrogênio acumuladas nas plantas em resposta a aplicação simultânea de Rhizobium sp. e Bacillus sp. nas sementes.

\section{Fungos Promotores de Crescimento Vegetal}

Os fungos, principalmente, os do gênero Trichoderma sp. exibem um papel consolidado na produção agrícola, devido a sua capacidade de colonizar a rizosfera e outros locais na parte aérea das plantas, promovendo efeitos benéficos no desenvolvimento vegetal (Mayo Pietro et al., 2020).

Trichoderma são fungos de vida livre que exibem a capacidade de atuarem em diversos mecanismos de controle biológico como: (a) parasitismo - relação nutricional entre dois seres vivos em que um dos componentes da relação, o parasita obtém todo ou parte de seu alimento às custas do outro componente, o hospedeiro; (b) hiperparasitismo - nível mais elevado de parasitismo, no qual o hospedeiro é também um parasita; (c) antibiose - interação na qual um ou mais metabólitos produzidos 
por um organismo têm efeito danoso sobre o outro; (d) competição - processo referente à interação entre dois ou mais organismos, empenhados na mesma ação e (e) indução de resistência de plantas às doenças - aumento da capacidade de defesa da planta contra amplo espectro de organismos fitopatogênicos (Guimarães et al., 2018).

Além do biocontrole mediado pelo contato físico com um outro microrganismo, o Trichoderma também libera diversos metabólitos voláteis e não voláteis (limita o crescimento do patógeno, ou da produção de metabólitos tóxicos, limitando assim o desenvolvimento do patógeno, da doença e favorecendo o desenvolvimento da planta hospedeira) (Cadore et al., 2018). Outro mecanismo observado neste gênero e capacidade de degradar paredes celular dos patógenos, alimentando-se do produto degradado e avançando rapidamente com o processo de colonização do patógeno (Guimarães et al., 2018).

Vários estudos mostram que Trichodrerma também atua como microrganismo promotor de crescimento vegetal, uma vez que ele é capaz de produzir fitormônios, solubilizar fosfatos e outros minerais (França et al., 2017), melhorar a eficiência do uso de nitrogênio, quando em combinação com outras espécies como Bacillus sp ou Rhizobium sp, e induzir defesa contra estresses bióticos e abióticos, principalmente, o estresse salino (Rubio et al., 2017).

Estudos recentes demonstram efeitos benéficos do Trichoderma sp. no aumento da porcentagem de germinação, diâmetro do hipocótilo, comprimento do sistema radicular na cultura do feijão (Mayo Pietro et al., 2020), no acúmulo de biomassa na cultura do arroz e milho (Chagas et al., 2017), aumento da produção de ácido indol-3-acético e solubilização de fosfato na cultura do tomate (França et al., 2017) e na supressão da Sclerotinia sclerotiorum na cultura da soja (Macena et al., 2020).

\section{Mecanismos de Ação dos Microrganismos Multifuncionais}

\subsection{Fixação biológica de nitrogênio}

A fixação biológica de nitrogênio (FBN) é considerada após a fotossíntese, o mais importante processo biológico para as plantas e foi o primeiro mecanismo de ação das RPCVs a ser descoberto (Elmerich, 2015). Segundo Chang et al. (2015), a FBN tem grande relevância econômica e ambiental, uma vez que as bactérias fixadoras de $\mathrm{N}$ apresentam grande eficiência (principalmente para a cultura da soja) e o N é o principal nutriente mineral limitante do crescimento vegetal (Vicente \& Dean, 2017).

O N é um nutriente fundamental para a síntese de biomoléculas como proteínas, ácidos nucleicos e inúmeros metabólitos primários e secundários essenciais para o adequado funcionamento das plantas (Chang et al., 2015). Embora a molécula de nitrogênio, $\mathrm{N}_{2}$, seja abundante na atmosfera, as plantas não são capazes de assimilá-la e, em decorrência disso, há a necessidade da adubação nitrogenada (Vicente \& Dean, 2017). A aplicação de fertilizante nitrogenado vem sendo a forma mais utilizada desde a década de 60; no entanto, com a descoberta das RPCVs diazotróficas, ou seja, aquelas que realizam FBN convertendo o $\mathrm{N}_{2}$ em sua forma amoniacal $\left(\mathrm{NH}_{3}\right)$, novos rumos nas práticas agrícolas estão sendo traçados (Elmerich, 2015). Com conversão o $\mathrm{N}_{2} \mathrm{em}$ sua forma amoniacal $\left(\mathrm{NH}_{3}\right)$, o $\mathrm{N}$ se torna biodisponível para a absorção vegetal (Zgadzaj et al., 2016) (Figura 3). Existe uma diversidade de RPCVs diazotróficas conhecidas, mas a base do processo de FBN é comum a todas elas por meio da ação da enzima nitrogenase, composta por proteínas codificadas pelo grupo de "operons" nif (Angel et al., 2018). 
Figura 3. Processo de nodulação de rizóbios em raízes de leguminosas - planta hospedeira.

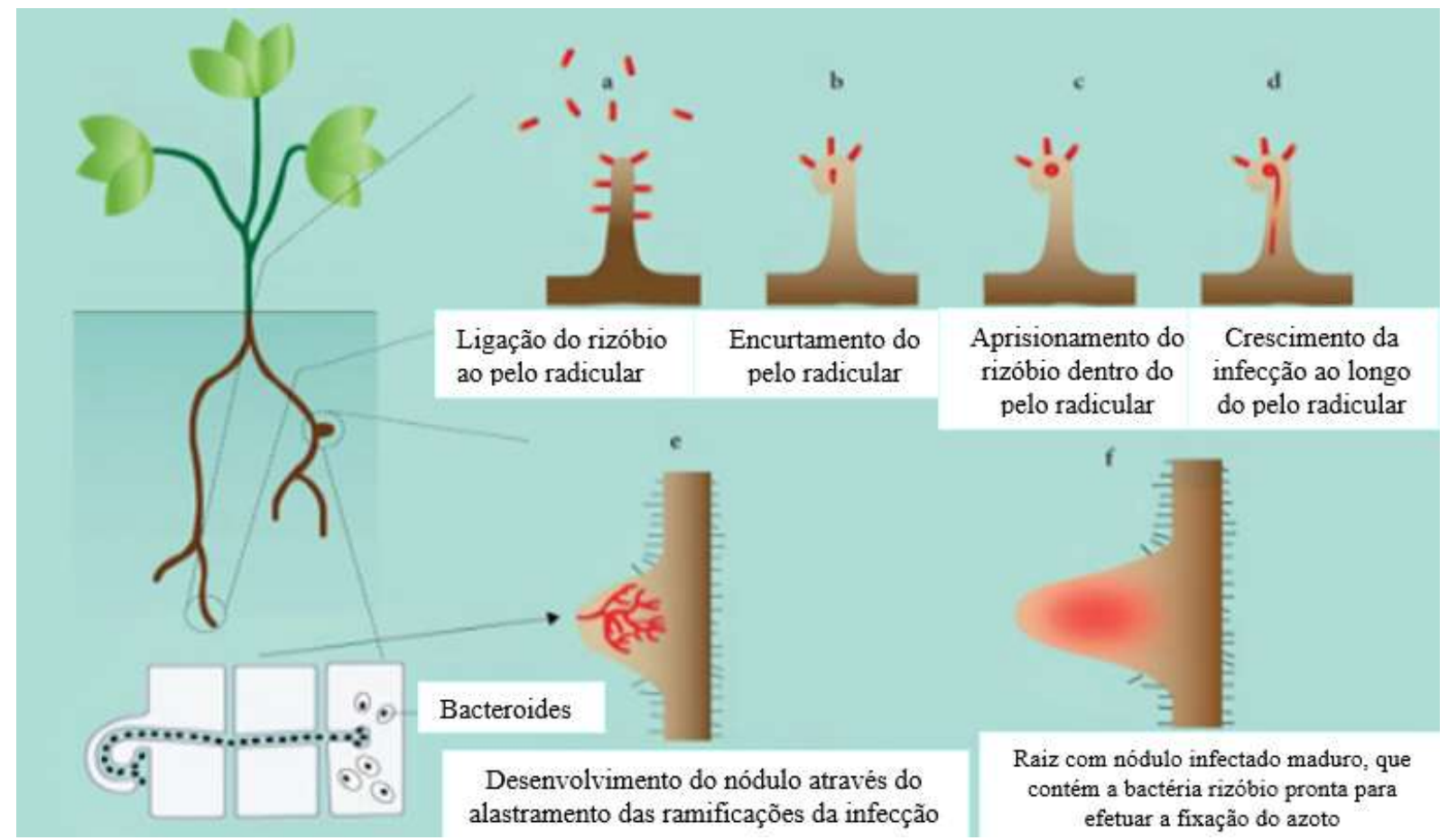

Fonte: Fernandes e Rodrigues (2014).

As principais bactérias fixadoras de $\mathrm{N}$ são as que estabelecem associação simbiótica com as leguminosas (todas as espécies do grupo iRPCV, que formam os nódulos), principalmente os gêneros Rhyzobium spp. e Bradyrhizobium spp. No entanto, algumas espécies de eRPCV de vida livre ou endofíticas associadas a gramíneas também podem promover FBN, mas de maneira menos eficiente do que as iRPCV, como as espécies do gênero Azospirillum sp. (Chang et al., 2015).

A prática de inoculação de RPCVs fixadoras de nitrogênio em culturas de interesse econômico, especialmente soja e feijão, com o propósito de suplementação nitrogenadas, já é bem estabelecida, com inúmeras linhagens comerciais disponíveis, principalmente de rizóbios (Baldani et al., 2014). No entanto são necessários mais estudos em relação a capacidade das eRPCV em promover a absorção de $\mathrm{N}$ em diferentes culturas, ou em auxiliar as iRPCV em aumentar a eficiência da FBN nas leguminosas, gramíneas dentre outras culturas, como espécies do gênero Azospirillum spp., que são bactérias diazotróficas (Chang et al., 2015). Aung et al. (2013) registraram aumento de 20 nódulos por planta de soja com a coinoculação de $B$. japonicum com Azospirillum sp. quando comparado a inoculação tradicional com somente B. japonicum.

\subsection{Solubilização de fosfato}

O fósforo é o segundo nutriente mais limitante ao crescimento das plantas, sendo componente estrutural de muitas coenzimas, fosfoproteínas, fosfolipídeos, ácidos nucléicos. Portanto, é um nutriente associado a vários processos fisiológicos primários como a fotossíntese, respiração e formação da membrana (Baby et al., 2016).

A solubilização de fosfato inorgânico é uma característica das bactérias independentemente de estar em interação com a planta. As rizobactérias e alguns fungos, incluindo o gênero Trichoderma sp. possuem papel fundamental na solubilização de fósforo não-lábil, apresentando-se como bioinsumo, alternativa ao uso de fertilizantes fosfatados sintéticos (Awais et al., 2017). O principal mecanismo de ação na solubilização de fosfatos inorgânicos é através dos ácidos orgânicos sintetizados por microrganismos, como por exemplo ácido glucônico, o que também promove a acidificação da célula microbiana e o ambiente ao seu redor (Fabianska et al., 2019). 
Além dos ácidos orgânicos, ocorrem também a liberação de prótons $\mathrm{H}^{+}$, a produção de substâncias quelantes, ou a produção de ácidos inorgânicos (Gupta et al., 2015). A liberação de prótons $\mathrm{H}^{+}$para a superfície celular externa ocorre em pelos processos de absorção de cátions ou com ainda pelo auxílio de ATPases. A produção de substâncias quelantes, ou a produção de ácidos inorgânicos, são mecanismos alternativos para a solubilização de fosfatos inorgânicos (Saber et al., 2009). Dentre os gêneros bacterianos solubilizadores de fósforo não-lábil citam-se: Agrobacterium, Arthrobacter, Azotobacter, Bacillus, Beijerinckia, Burkholderia, Enterobacter, Erwinia, Flavobacterium, Microbacterium, Pseudomonas, Rhizobium, Rhodococcus e Serratia (Bhattacharyya \& Jha, 2012).

Por outro lado, a solubilização de fosfatos orgânicos, também chamada mineralização, ocorre a partir de restos vegetais e animais contendo elevados teores de compostos fosfatados (Ahemad \& Kibret, 2014). Numerosas espécies saprofíticas realizam a decomposição da matéria orgânica do solo, que liberam o radical ortofosfato da estrutura de carbono da molécula, por processos de respiração e sob forte influência de fatores ambientais (Marra et al., 2012). As principais moléculas fornecedoras de $\mathrm{P}$ depois da mineralização são os ácidos nucléicos, fosfolipídeos e açúcares fosfatados e são considerados facilmente degradados, já os ácidos fítico, polifosfatos e fosfonatos são mineralizados lentamente (Baby et al., 2016).

Esta mineralização é promovida por enzimas chamadas fosfatases, ou fosfohidrolases, classificadas em ácidas ou alcalinas de acordo com o pH ótimo de atividade. Estas enzimas podem ser secretadas fora da membrana plasmática, ou permanecerem retidas na membrana como proteínas solúveis (Teymouri et al., 2016). Desta forma, microrganismos capazes de solubilizar e mineralizar fosfatos podem promover a utilização dos fosfatos naturais do solo, beneficiando as culturas, diminuindo os custos de produção e aumentando a eficiência na adubação (Fabiańska et al., 2019).

Machado (2015), detectou linhagens bacterianas endofíticas do gênero Bacillus e Citrobacter, isoladas da cultura de pinhão-manso, com potencial de solubilizar fosfato. Chauhan et al. (2015) trabalhando com bactérias isoladas de colmos de cana-de-açúcar, relataram a presença de quatro linhagens do gênero Pseudomonas e Bacillus, solubilizadoras de fósforo nãolábil que afetaram positivamente o desenvolvimento das plantas, evidenciando aumento da germinação (55\%), número de colmos (20\%), altura (18\%), circunferência e peso de colmos (8 e 51\%, respectivamente), produtividade (39\%) e porcentagem de açúcares disponíveis (6\%) em relação ao tratamento controle. Durán et al. (2016) inocularam Bacillus sp. e Klebsiella sp. em alface e mostraram maiores teores de fósforo, potássio e biomassa seca na parte aérea em plantas inoculadas quando comparadas às não inoculadas.

\subsection{Produção de hormônios}

Os hormônios vegetais são mensageiros químicos que influenciam a capacidade da planta de reagir ao ambiente (Enders \& Strader, 2015). Estes são compostos orgânicos naturais que são eficazes em concentrações muito baixas e são principalmente sintetizados em certas partes da planta e transportados para outro local. Os hormônios vegetais, também chamados de fitormônios, influenciam os processos fisiológicos tais como crescimento, diferenciação, desenvolvimento; bem como movimento estomático (Sureshbabu et al., 2016). Ressalta-se que a resposta de cada planta geralmente é o resultado de dois ou mais hormônios agindo em paralelo (Kang et al., 2019). Por outro lado, as RPCVs e fungos do gênero Trichoderma sp. também produzem fitohormônios que podem influenciar os seus próprios processos fisiológicos como também os das plantas e, por isso, passam a ser denominados reguladores de crescimento das plantas (Singh et al., 2017).

Microrganismos multifuncionais, em sua maioria, produzem hormônios como auxinas, citocininas, giberelinas, ácido abscísico e etileno, e mais recentemente estrigolactonas e brassinosteroides que auxiliam o desenvolvimento vegetal (Sureshbabu et al., 2016). Auxinas são uma classe hormonal responsável em aumentar a formação de primórdios radiculares (Taiz \& Zeiger, 2004). Dentre os gêneros conhecidos por expressar esse mecanismo citam-se Arthrobacter, Azospirillum, Bradyrhizobium, Bacillus, Pseudomonas e Paenibacillus (Singh et al., 2017). 
De acordo Vejan et al. (2016), um microrganismo que possui a capacidade de produzir ou alterar a concentração de reguladores de crescimento vegetal como AIA, GA, citocininas e etileno é denominado fitoestimulador. A aplicação de RPCVs, produtoras de hormônios, pode ser útil para melhorar a fisiologia, biomassa e rendimento da cultura, aumentando o tamanho, número de ramos e área de superfície das raízes (Enders \& Strader, 2015). Aumentos na área de superfície da folha facilitam a absorção da luz solar, enquanto o aumento da ramificação da raiz e da área de superfície facilitam a absorção de água e nutrientes (Kang et al., 2019). Araújo et al. (2014) observaram efeito significativo na altura, massa seca de parte aérea e sistema radicular em mudas micropropagadas de bananeira, tratadas com rizobactérias produtoras de AIA.

\subsection{Produção de sideróforos}

O ferro é um micronutriente essencial para as plantas e está envolvido em vários processos fundamentais como fotossíntese, respiração, síntese de DNA e hormônios (Puig et al., 2017). Ele atua como um catalisador na síntese de clorofila (Hu et al., 2017), processos enzimáticos envolvendo oxigênio (peroxidase, catalase), hidrogênio (hidrogenases), nitrogênio (nitrogenases) e processos de transferência de elétrons nos sistemas respiratório e fotossintético (Masepohl, 2017).

Embora amplamente disponível na natureza, o Fe nem sempre é acessível às plantas e muitas vezes limita a produtividade biológica de plantas (Briat et al., 2015). Assim, as plantas desenvolveram diferentes estratégias para combater esta limitação como a liberação de prótons e ácidos orgânicos pelas raízes para diminuir o pH do solo e aumentar assim a disponibilidade de ferro e/ou a liberação de moléculas de baixo peso molecular (sideróforos) que se ligam ao mineral ( $\mathrm{Fe}$ ) e, em seguida, são absorvidas pelas células da raiz (Johnstone \& Nolan, 2015). Assim como as plantas, as rizobactérias e fungos do gênero de Trichoderma sp. podem produzir uma ampla gama de sideróforos atuando como agentes quelantes de ferro a partir de compostos minerais ou orgânicos em condições de limitação do elemento (Huo et al., 2020).

Sideróforos são moléculas sequestradoras de ferro de baixo peso molecular (400 a 1000 Daltons) e elevada afinidade pelo substrato (Kd $10^{-20}$ a $10^{-50}$ ), secretadas por microrganismos em resposta a baixa disponibilidade de $\mathrm{Fe}^{+3}$ em solução (Briat et al., 2015). Estes compostos atuam do lado externo da membrana celular, capturando moléculas de ferro em solução e ligando-se especificamente a receptores do complexo localizados na membrana, por onde são absorvidos, tornando assim o ferro absorvido disponível para o crescimento dos vegetais (Masepohl, 2017) (Figura 4). 
Figura 4. Mecanismo de produção de sideróforos pelos microrganismos que se ligam a moléculas de Fe.

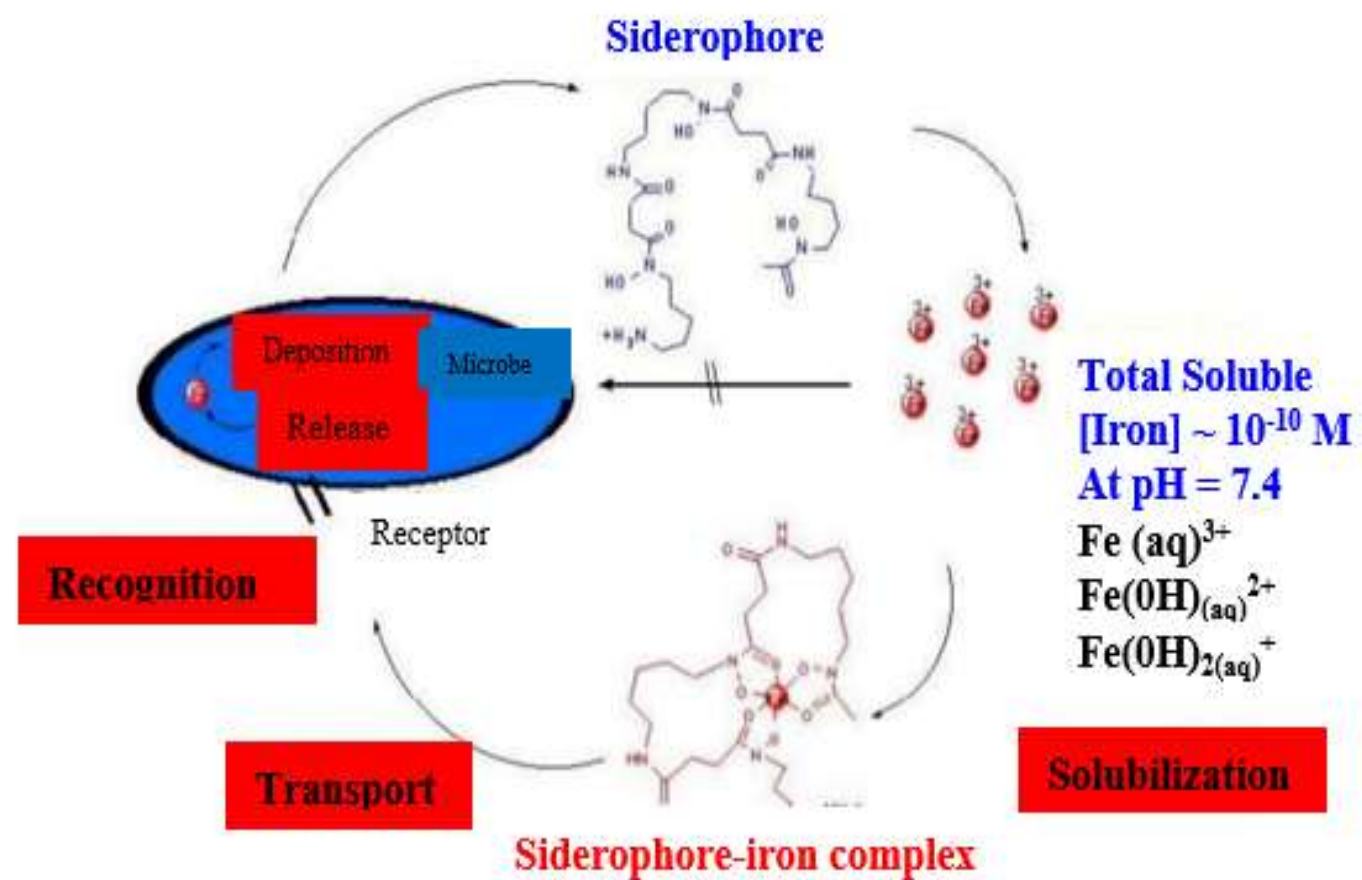

Fonte: Serrano (2009).

Além da disponibilização de ferro para o desenvolvimento da bactéria, a formação do complexo ferro-sideróforo também impede a proliferação de patógenos devido à competição pelo nutriente no ambiente (Puig et al., 2017). Certas espécies do gênero Pseudomonas produzem sideróforos que quelam o ferro na rizosfera, inibindo o desenvolvimento de alguns microrganismos que dependem do ferro para sua sobrevivência, incluindo fitopatógenos, que têm menor capacidade de competição por ferro (Johnstone \& Nolan, 2015).

Dimkpa et al. (2009) mostraram que sideróforos possibilitam a captação de Fe pelas plantas ao invés de outros minerais como $\mathrm{Al} \mathrm{Cu}, \mathrm{Mn}$ e $\mathrm{Ni}$ em altas concentrações na solução do solo. Por outro lado, na ausência de sideróforos, esses metais inibiam a absorção de Fe para o feijão-caupi (Vigna unguiculata). Rajkumar e Freitas (2008) verificaram que a produção de sideróforos por Pseudomonas sp. e Bacillus megaterium, em conjunto como outros mecanismos, como a produção de hormônios e a ação da ACCdesaminase, contribuíram para o incremento da produtividade em plantas de mostarda marrom (Brasica juncea).

\subsection{Produção de biofilme}

O biofilme pode ser definido como uma comunidade microbiana altamente aderente entre si ou a uma superfície, sendo composta por carboidratos extracelulares, proteínas, exopolissacarídeos e até mesmo DNA (Branda et al., 2005). As rizobactérias e algumas gêneros de fungos são conhecidas por formarem biofilme para auxiliar sua sobrevivência em várias condições de estresse: resistência a antibióticos, proteção contra fitopatógenos e dessecação e agentes estressantes antimicrobianos (Parsek \& Fuqua, 2004). Kavamura et al. (2013) observaram a produção de biofilme pelas bactérias do gênero Bacillus, isoladas de cactáceas do semiárido brasileiro, sob condições estressantes. Timmusk (2005) mostrou que Paenibacillus também é capaz de colonizar raízes de plantas de trigo, cevada, alho e feijão-verde e formar biofilme.

Durante o crescimento de uma planta, o biofilme formado em decorrência da interação microrganismos-planta pode auxiliar na colonização radicular (Ramey et al., 2004), proteger as células da privação de nutrientes, fagocitose e alterações de pH e, através da secreção de substâncias polissacarídicas, conferir estabilidade às plantas em condições limitantes de água 
(Kasim et al., 2016). Os exopolissacarídeos formadores de biofilme são altamente hidratados e conseguem reter alta quantidade de água no interior de suas moléculas, conferindo-lhes característica hidrofílica, e portanto, com alta capacidade de absorção e retenção de água, que ao habitarem a região da rizosfera das plantas, conseguem proteger as raízes da secagem do solo (Rolli et al., 2014).

\subsection{Controle biológico}

As bactérias e fungos desempenham a função de agentes biológicos induzindo a resistência sistêmica em plantas, produzindo antibióticos e sideróforos que inibem o crescimento dos patógenos (Pinho et al., 2020). No contexto da resistência sistêmica, os hormônios produzidos por bactérias podem atuar como estimulantes do sistema imune das plantas, interferindo no funcionamento dos principais sistemas de defesa vegetal. Dentre estes, destacam-se a resistência sistêmica adquirida (SAR) e a resistência sistêmica induzida (ISR) (Oliveira et al., 2017).

Resistência sistêmica adquirida (SAR) é um sistema de largo espectro que depende do hormônio ácido salicílico e não apresenta especificidade à infecção inicial, tendo efeito, principalmente, contra patógenos biotróficos (Neugebauer et al., 2019). Geralmente, a capacidade de defesa da planta é adquirida após a primeira infecção por um microrganismo patogênico, que leva a uma morte celular programada através, iniciando uma cascata de reações que resultam na secreção (Rocha \& Moura, 2013). A ação se inicia em um sistema de defesa localizado chamado de hipersensibilidade, submerge a acumulação de fitoalexinas e requerimento da síntese de aceleração de enzimas (enilalanina, amonialiase, calcone sintase, peroxidase e proteínas) associadas com a biossíntese fenólica (Pinho et al., 2020).

Resistência sistêmica induzida (ISR) é um sistema dependente do ácido jasmônico e da sinergia com o etileno, sendo ativada por microrganismos patogênicos (Neugebauer et al., 2019). Estudos recentes mostraram efeitos benéficos de rizobactérias na redução da população de nematoides de galha (Meloigogyne spp.) no feijão-comum (Oliveira et al., 2017), no controle de fungos e na qualidade sanitária de sementes de arroz, eficácia na inibição do crescimento micelial de Sclerotinia sclerotiorum e produção de compostos orgânicos voláteis no controle do patógeno na cultura da soja (Pinho et al., 2020). Adicionalmente, atuam na resiliência dos sistemas de cultivo e na indução de resistência a doença (Nascente et al., 2017).

Os mecanismos de ação do Trichoderma sp. no controle biológico de fitopatógenos e na promoção de crescimento vegetal são variados (Neugebauer et al., 2019). O Trichoderma sp. possui característica hiperparasitária, pois pode detectar e localizar hifas de fungos suscetíveis, crescendo em sua direção, presumivelmente em resposta a estímulos químicos produzidos pela hifa hospedeira, formar estruturas semelhantes a opressórios e enrolar-se fortemente em toda a sua extensão para, então, penetrar e digerir a hifa, isso denomina-se micoparasitismo e ressalta ser um importante mecanismo de ação de biocontrole (Machado et al., 2012).

Dentre as substâncias que podem ser sintetizadas, muitas espécies de Trichoderma já estudadas produzem metabólitos secundários tóxicos, como antibióticos e enzimas líticas capazes de inibir e destruir propágulos de fungos fitopatogênicos (Chagas et al., 2017). A indução de resistência por Trichoderma sp. é outro mecanismo de ação que vem sendo pesquisado (Chen et al., 2015). Além disso, as plantas levadas ao estado de indução apresentam aumento nas atividades de enzimas, tais como, quitinases, glucanases e peroxidases, envolvidas nas rotas de percepção da presença de patógenos em potencial e nas rotas de sinalização bioquímica a pontos distantes do sítio onde o sinal foi originado. Por consequência, verifica-se um aumento nas atividades de enzimas envolvidas na síntese de componentes de resistência (Silva et al., 2018).

Estudos evidenciaram a ação de microrganismos como o Trichoderma sp. no potencial antagônico contra Fusarium moniliforme na cultura do milho (Medeiros et al., 2020), no controle de nematoide Pratylenchus brachyurus na cultura da soja (Oliveira et al., 2021), promoção de crescimento com presença de Rhizoctonia solani no feijão-comum (Mayo Pietro et al., 2020). 


\section{Considerações Finais}

A previsão de aumento populacional pressiona o cenário agropecuário por alternativas para suprir a necessidade alimentícia da população mundial. Os avanços tecnológicos advindos da industrialização e da era digital são os grandes responsáveis pelo impulso científico e tecnológico que alavanca a agricultura e a produção agrícola. Mas, por outro lado, cria uma tensão na sustentabilidade das práticas agrícolas para a produção de alimentos.

O mundo enfrenta colapsos ambientais e o cenário mercadológico atual tende pela procura de alimentos produzidos de forma sustentável. E, nesse sentido, os bioinsumos são vistos como tecnologias sustentáveis que atuam de forma intrínseca e extrínseca sobre as plantas contribuindo para o incremento produtivo e a estabilidade do equilíbrio do agroecossistema.

\section{Referências}

Abhilash, P. C. C., Dubey, R. K., Tripathi, V., Gupta, V. K. \& Singh, H. B. (2016). Plant Growth-Promoting Microorganisms for Environmental Sustainability. Trends in Biotechnology, 34 (11), 847-850.

Ahemad, M. S. \& Kibret, M. (2014). Mechanisms and applications of plant growth promoting rhizobacteria: Current perspective. Journal of King Saud University - Science, 26 (1), 1-20.

Akhtar, N., Mujeeb, F., Qureshi, M. A., Rafique, M., Riaz, A. \& Ali, M. A. (2014). Phosphate solubilizing potential of Rhizobium and Bacillus species for enhancing yield and available phosphorus in maize crop (Zea mays). International Journal of Agronomy and Agricultural Research, 4 (1), 58-66.

Angel, R., Nepel, M., Panhölzl, C., Schmidt, H., Herbold, C. W., Eichorst, S. A. \& Woebken, D. (2018). Evaluation of Primers Targeting the Diazotroph Functional Gene and Development of NifMAP - A Bioinformatics Pipeline for Analyzing nifH Amplicon Data. Frontiers in Microbiology, 9 (703), 1-15.

Araújo, K. S., Cardoso, K. G. V., Peixoto, C. C., Ramos, E., Silva, H. S. A. \&Trindade, A. V. (2014). Promoção do crescimento em mudas micropropagadas de bananeira por rizobactérias. https://www.embrapa.br/busca-de-publicacoes/-/publicacao/873156/promocao-do-crescimento-em-mudas-micropropagadasde-bananeira-por-rizobacterias

Aung, T. T. (2013). Enhanced soybean biomass by co-inoculation of Bradyrhizobium japonicum and plant growth promoting rhizobacteria and effects on microbial community structures. African Journal of Microbiology Research, 7 (29), 3858- 3873.

Awais, M., Tariq, M., Ali, A., Ali, Q., Khan, A., Tabassum, B., Nasir, I. A. \& Husnain, T. (2017). Isolation, characterization and inter-relationship of phosphate solubilizing bacteria from the rhizosphere of sugarcane and rice. Biocatalysis and Agricultural Biotechnology, 11 (1), $312-321$.

Baby, K., Kumar, A. \& Mallick, M. A. (2016). Phosphate solubilizing microbes: an effective and alternative approach as biofertilizers. International Journal Pharm Science, 8 (2), 37-40.

Baldani, J. I., Reis, V. M., Videira, S. S., Boddey, L. H. \& Baldani, V. L. D. (2014). The art of isolating nitrogen-fixing bacteria from non-leguminous plants using N-free semisolid media: a practical guide for microbiologists. Plant and Soil, 384 (1), 413-431.

Bashan, Y., Kamnev, A. A. \& Bashan, L. E. (2013). Tricalcium phosphate is inappropriate as a universal selection factor for isolating and testing phosphatesolubilizing bacteria that enhance plant growth: A proposal for an alternative procedure. Biology and Fertility of Soils, 49 (1), $465-479$.

Bhattacharyya, P. N. \& Jha, D. K. (2012). Plant growth-promoting rhizobacteria (PGPR): emergence in agriculture. World Journal of Microbiology and Biotechnology, 28 (4), 1327-1350.

Branda, S. S., Vik, S., Friedman, L. \& Kolter, R. (2005). Biofilms: the matrix revisited. Trends in Microbiology, 13 (1), $20-26$.

Briat, J. F., Dubos, C. \& Gaymard, F. (2015). Iron nutrition, biomass production, and plant product quality. Trends in Plant Science, 20 (1), 33-40.

Brito, D. (2020). Mercado de biodefensivos cresce mais de $70 \%$ no Brasil em um ano. Ministério da Agricultura, Pecuária e Abastecimento. https://www.gov.br/agricultura/pt-br/assuntos/noticias/feffmercado-de-biodefensivos-cresce-em-mais-de-50-no-brasil

Cadore, L. S., Vey, R. T., Fresinghelli, J. C. F., Dotto, L. \& Ethur, L. Z. (2018). Evaluation of initial growth of soybean using Trichoderma formulations. Enciclopédia Biosfera, 15 (27), 170-179.

Carvalho, T. \& Chagas, I. (2019). Brasil, campeão mundial em consumo de agrotóxicos. Politize. https://www.politize.com.br/brasil-campeao-mundial-emconsumo-de-agrotoxicos/

Chagas, L. F. B., Chagas Junior, A. F., Soares, L. P. \& Fidelis, R. R. (2017). Trichoderma na promoção do crescimento vegetal. Revista de Agricultura Neotropical, 4 (3), 97-102.

Chang, W. S., Lee, H. I. \& Hungria, M. (2015). Soybean Production in the Americas. Principles of Plant-Microbe Interactions: Microbes for Sustainable Agriculture, 393- 400 .

Chauhan, H., Bagyaraj, D. J., Selvakumar, G. \& Sundaram, S. P. (2015). Novel plant growth promoting rhizobacteria - Prospects and potential. Applied Soil Ecology, 95 (1), 38-53.

Chen, L., Jiang, H., Cheng, O., Chen, J., Wu, G., Kumar, A., Sun, M. \& Liu, Z. (2015). Enhanced nematicidal potential of the Chitinase pachi from Pseudomonas aeruginosa in association with Cry21Aa. Scientific Reports, 24 (5), 1-11. 
Dalolio, R. S., Borin, E., Cruz, R. M. S. \& Alberton, O. (2018). Co-inoculação de soja com Bradyrhizobium e Azospirillum. Journal of Agronomic Sciences, 7 (2), 1-7.

Dimkpa, C. O., Dirk, M., Ales, S., Georg, B. \& Erika, K. (2009). Metal-induced oxidative stress impacting plant growth in contaminated soil is alleviated by microbial siderophores. Soil Biology Biochemistry, 41 (1), 154-162.

Durán, P., Acuña, J. J., Armada, E., López Castillo, O. M., Cornejo, P., Mora, M. L. \& Azcón, R. (2016). Inoculation with selenobacteria and arbuscular mycorrhizal fungi to enhance selenium content in lettuce plants and improve tolerance against drought stress. Journal of Soil Science and Plant Nutrition, 16 (1), 201-225.

Elmerich, C. (2015). One Hundred Years Discovery of Nitrogen-Fixing Rhizobacteria Biological Nitrogen Fixation. John Wiley \& Sons, 897-912.

Enders, T. A. \& Strader, L. C. (2015). Auxin activity: Past, present, and future. American Journal of Botany, 10 (2), $180-196$.

Fabiańska, I., Gerlach, N., Almario, J. \& Bucher, M. (2019). Plant-mediated effects of soil phosphorus on the root-associated fungal microbiota in Arabidopsis thaliana. New Phytologist, 221 (4), 2123-2137.

França, D. V. C., Kupper, K. C., Magri, M. M. R., Gomes, T. M. \& Rossi, F. (2017). Trichoderma spp. isolates with potential of phosphate solubilization and growth promotion in cherry tomato. Pesquisa Agropecuária Tropical, 47 (4), 360-368.

González, C. R., Aguilar, L. M., Trujillo, A. R., Santos, P. E., Mellado, C. J., Santos, P. \& Mellado, J. C. (2011). High diversity of culturable Burkholderia species associated with sugarcane. Plant and Soil, 345 (1), 155-169.

Guimarães, G. R., Pereira, F. T., Mello, S. C. M. \& Carvalho, D. D. C. (2018). Employment of Trichoderma to control Cladosporium sp. and Sclerotinia sclerotiorum and bean growth promoting in Brazil. Caderno de Pesquisa, 30 (2), 28-37.

Gupta, G., Parihar, S. S., Ahirwar, N. K., Snehi, S. K. \& Singh, V. (2015). Plant Growth Promoting Rhizobacteria (PGPR): Current and Future Prospects for Development of Sustainable Agriculture. Journal of Microbiol \& Biochemical Technology, 7 (2), 96-102.

Hu, X., Page, M. T., Sumida, A., Tanaka, A., Terry, M. J. \& Tanaka, R. (2017). The iron-sulfur cluster biosynthesis protein sufb is required for chlorophyll synthesis, but not phytochrome signaling. The Plant Journal, 89 (6), 1184-1194.

Huo, Y., Kang, J. P., Ahn, J. C., Kim, Y. J., Piao, C. H., Yang, D. U. \& Yang, D. C. (2020). Siderophore-producing rhizobacteria reduce heavy metal-induced oxidative stress in Panax ginseng Meyer. Journal of Ginseng Research, 1226-1243.

Johnstone, T. C. \& Nolan, E. M. (2015). Beyond iron: non-classical biological functions of bacterial siderophores. Dalton Transactions, 44 (14), $6320-6339$.

Kang, S. M., Khan, A. L., Wagas, M., Asaf, S., Lee, K. E., Park, Y. G., Kim, A. Y., Khan, M. A., You, Y. H. \& Lee, I. J. (2019). Integrated phytohormone production by the plant growth-promoting rhizobacterium Bacillus tequilensis SSB07 induced thermotolerance in soybean. Journal of Plant Interactions, 14 (1), 416-423.

Kasim, W. A., Gaafar, R. M., Abou-Ali, R. M., Omar, M. N. \& Hewait, H. M. (2016). Effect of biofilm forming plant growth promoting rhizobacteria on salinity tolerance in barley. Annals of Agricultural Sciences, 61 (2), 217-227.

Kavamura, V. N., Santos, S. N., Silva, J. L., Parma, M. M., Avila, L. A. (2013). Screening of Brazilian cacti rhizobacetria for plant growth promotion under drought. Microbiological Research, 168 (4), 183-191.

Liotti, R. G., Figueiredo, M. I. S., Silva, G. F., Mendonça, E. A. F. \& Soares, M. A. (2018). Diversity of cultivable bacterial endophytes in Paullinia cupana and their potential for plant growth promotion and phytopathogen control. Microbiological Research, 207 (1), 8-18.

Lopes, R., Tsui, S., Gonçalves, P. J. R. O. \& Queiroz, M. (2020). A look into a multifunctional toolbox: endophytic Bacillus species provide broad and underexploited benefits for plants. World Journal of Microbiology and Biotechnology, 34 (7), 94-102.

Macena, A. M. F., Kobori, N. N., Mascarin, G. M., Vida, J. B. \& Hartman, G. L. (2020). Antagonism of Trichoderma-based biofungicides against Brazilian and North American isolates of Sclerotinia sclerotiorum and growth promotion of soybean. BioControl, 65 (1), $235-246$.

Machado, P. C. (2015). Identificação molecular e caracterização bioquímica de bactérias endofíticas associadas à cultura do pinhão-manso (Jatropha curcas L.) com potencial biotecnológico. (Dissertação em Biotecnologia). Universidade Federal de São Carlos.

Machado, D. F. M., Parzianello, F. R., Silva, A. C. F., Antoniolli, Z. I. (2012). Trichoderma no Brasil: o fungo e o bioagente. Revista de Ciências Agrárias, 35 (1), 274-288.

Marra, L. M., Soares, C. R. F. S., Oliveira, S. M., Ferreira, P. A. A., Soares, B. L., Carvalho, R. F., Lima, J. M. \& Moreira, F. M. S. (2012). Biological nitrogen fixation and phosphate solubilization by bacteria isolated from tropical soils. Plant and Soil, 357 (1), $289-307$.

Martínez Viveros, O., Jorquera, M. A., Crowley, D. E., Gajardo, G. \& Mora, M. L. (2010). Mechanisms and practical considerations involved in plant growth promotion by Rhizobacteria. Journal of Soil Science and Plant Nutrition, 10 (3), 293-319.

Masepohl B. (2017). Modern topics in the phototrophic prokaryotes: Metabolism, bioenergetics, and omics. Springer: Cham.

Mayo Pietro, S., Campelo, M. P., Lorenzana, A., Rodríguez González, A., Reinoso, B., Gutiérrez, S. \& Casquero, P. A. (2020). Antifungal activity and bean growth promotion of Trichoderma strains isolated from seed vs soil. European Journal of Plant Pathology, 158, 817-828.

Medeiros, J. C. D., Martins, W. S. \& Miranda, F. F. R. (2020). Antagonism of Trichoderma spp. in Fusarium moniliforme biocontrol in corn culture. Revista Sítio Novo, 4 (4), 169-178. 
Montaldo, Y. C. (2016). Bioprospecção e isolamento de bactérias associadas à cana-de-açúcar (Saccharum officinarum L.) com características para a promoção de crescimento vegetal. Tese (Doutorado em Rede Nordeste de Biotecnologia) - Instituto de Química e Biotecnologia, Programa de Pós Graduação em Rede Nordeste de Biotecnologia, Universidade Federal de Alagoas, Maceió, 101.

Moreira, A. L. L. \& Araújo, F. F. (2013). Bioprospecção de isolados de Bacillus spp. como potenciais promotores de crescimento de Eucalyptus urograndis. Revista Árvore, 37 (5), 933-943.

Nascente, A. S., Fillipi, M. C., Lanna, A. C., Souza, V. L., Silva, L. \& Silva, G. B. (2017). Biomass, gas exchange, and nutrient contents in upland rice plants affected application forms of microorganism growth promoters. Environmental Science Pollution Research, 24, $2956-2965$.

Neugebauer, W. C., Gomes, C. B. \& Mota, M. (2019). Seleção de bactérias para controle biológico de Meloidogyne incognita em figueira. Revista de la Facultad de Agronomía, 118 (1), 51-60.

Oliveira, C. M., Almeida, N. O., Cortes, M. V. C. B., Lobo Júnior, M., Rocha, M. R. \& Ulhoa, C. J. (2021). Biological control of Pratylenchus brachyurus with isolates of Trichoderma spp. on soybean. Biological Control, 152.

Oliveira, G. R. F., Silva, M. S., Proença, S. L., Bossolani, J. W., Camargo, J. A., Franco, F. S. \& SÁ, M. E. (2017). Influence of Bacillus subtilis in nematodes biological control and production aspects of bean. Brazilian Journal of Biosystems Engineering, 11 (1), 47-58.

Parsek, M. R. \& Fuqua, C. (2004). Biofilms: emerging themes and challenges in studies of surface-associated microbial life. Journal of Bacteriology, 186 (14), 4427-4440.

Pereira A. S., Shitsuka, D. M., Parreira, F. J. \& Shitsuka, R. (2018). Metodologia da pesquisa científica. UFSM.https://repositorio.ufsm.br /bitstream/handle/1/15824/Lic_Computacao_Metodologia-Pesquisa-Cientifica.pdf?sequence=1

Pietro Souza, W., Campos Pereira, F., Mello, I. S., Stachack, F. F. F., Terezo, A. J., Cunha, C. N., White, J. F., Li, H. \& Soares, M. A. (2020). Mercury resistance and bioremediation mediated by endophytic fungi. Chemosphere, 240, 1-42.

Pinho, R. S. C., Pozzebon, B. C., Rodrigues, K. R. R., Arns, R. B., Alves, C. A. \& Bergmann, M. B. (2020). Rizobactérias no controle de Sclerotinia sclerotiorum, e efeitos no desenvolvimento vegetativo de plântulas de soja. Colloquium Agrariae, 16 (4), $110-120$.

Puig, S., Ramos Alonso, L., Romero, A. M. \& Martínez Pastor, M. T. (2017). The elemental role of iron in DNA synthesis and repair. Metallomics, 9 (11), 1483-1500.

Rai, P. K., Singh, M., Anand, K., Saurabh, S., Kaur, T., Kour, D., Yaday, A. N. \& Kumar, M. (2020). Role and potential applications of plant growthpromoting rhizobacteria for sustainable agriculture. Trends of Microbial Biotechnology for Sustainable Agriculture and Biomedicine Systems: Diversity and Functional Perspectives, 49-60.

Rajkumar, M. \& Freitas, H. (2008). Effects of inoculation of plant growth promoting bacteria on Ni uptake by Indian mustard. Bioresource Technology, 99 (9), 3491-3498.

Ramey, B. E., Koutsoudis, M., Von Bodman, S. B. \& Fuqua, C. (2004). Biofilm formation in plant-microbeassociations. Current Opinion in Microbiology, 7 (6), 602-609, 2004.

Ratz, R. J., Palácio, S. M., Espinoza, F. R., Vicentino, R. C., Michelim, H. J. \& Richter, L. M. (2017). Potencial biotecnológico de rizobactérias promotoras de crescimento de plantas no cultivo de milho e soja. Engevista, 19 (4), 890-905.

Rocha, D. J. A. \& Moura, A. B. (2013). Biological control of tomato wilt caused by Ralstonia solanacearum and Fusarium oxysporum f. sp. lycopersici by rhizobacteria. Tropical Plant Pathology, 38 (5), 423-430

Rolli, E., Marasco, R., Vigani, G., Ettoumi, B., Mapelli, F., Deangelis, M. L., Gandolfi, C., Casati, E., Previtali, F., Gerbino, R., Cei, F. P., Borin, S., Sorlini, C., Zocchi, G. \& Daniele, D. (2014). Improved plant resistance to drought is promoted by the root-associated microbiome as a water stress-dependent trait. Environmental Microbiology, 17, 316-331.

Rout, M. E. (2014). The Plant Microbiome. Advances in Botanical Research. Academic Press, 69, 279-309.

Rubio, M. B., Hermosa, R., Vicente, R., Gómez-Acosta, F. A., Morcuende, R., Monte, E. \& Bettiol, W. (2017). The Combination of Trichoderma harzianum and Chemical Fertilization Leads to the Deregulation of Phytohormone Networking, Preventing the Adaptive Responses of Tomato Plants to Salt Stress. Frontiers in Plant Science, 8 (294), 1-14.

Saber, W. I. A., Ghanem, K. M. \& El Hersh, M. S. (2009). Rock phosphate solubilization by two isolates of Aspergillusniger and Penicillium sp. and their promotion to mung bean plants. Research Journal of Microbiology, 4 (1), 235-250.

Santoyo, G., Moreno Hagelsieb, G., del Carmen Orozco Mosqueda, M. \& Glick, B. R. (2016). Plant growth-promoting bacterial endophytes. Microbiological Research. 183, 92-99.

Schlaeppi, K. \& Bulgarelli, D. (2015). The Plant Microbiome at Work. Molecular Plant Microbe Interactions, 28 (3), $212-217$.

Silva, C. M., Pinheiro, C. C. C., Sousa, I. A. L., Lins, P. M. P., Silva, G. B. \& Carvalho, E. A. (2018). Biologic control of Bursaphelenchus cocophilus with rhizobacteria and Trichoderma isolates. Nativa, 6 (3), 233-240.

Silva, M. A., Nascente, A. S., Filippi, M. C. C., Lanna, A. C., Silva, G. B. \& Silva, J. F. A. (2020). Individual and combined growth-promoting microorganisms affect biomass production, gas exchange and nutrient content in soybean plants. Revista Caatinga, 33 (3), 619-632.

Singh, S., Singh, V. \& Pal, K. (2017). Importance of microorganisms in agriculture. Climate and Environmental changes: Impact, Challenges and Solutions, 1, 93-117. 
Research, Society and Development, v. 10, n. 2, e50810212725, 2021

(CC BY 4.0) | ISSN 2525-3409 | DOI: http://dx.doi.org/10.33448/rsd-v10i2.12725

Sousa, I. M., Nascente, A. S. \& Filippi, M. C. C. (2019). Bactérias promotoras do crescimento radicular em plântulas de dois cultivares de arroz irrigado por inundação. Colloquium Agrariae, 15 (2), 140-145.

Steffen, G. P. K., Maldaner, J., Missio, E. L. \& Steffen, R. B. (2018). Trichoderma controla fitonematóides e aumenta produtividade da soja, Campos \& Negócios. https://revistacampoenegocios.com.br/trichoderma-controla-fitonematoides-e-aumenta-produtividade-da-soja/

Sureshbabu, K., Amaresan, N. \& Kumar, K. (2016). Amazing multiple function properties of plant growth promoting rhizobacteria in the rhizosphere soil. International Journal of Current Microbiololy and Applied Sciences, 5 (2), 661-683.

Taiz, L. \& Zeiger, E. (2004). Fisiologia vegetal. Artmed.

Teymouri, M., Akhtari, J., Karkhane, M. \& Marzban, A. (2016). Assessment of phosphate solubilization activity of Rhizobacteria in mangrove forest. Biocatalysis and Agricultural Biotechnology, 5, 168-172.

Timmusk, S., Grantcharova, N. \& Wagner, E. G. H. (2005). Paenibacillus polymyxa invades plant roots and forms biofilms. Applied and Environmental Microbiology, 71 (11), 292-7300.

Vejan, P., Abdullah, R., Khadiran, T., Ismail, S. \& Boyce, A. N. (2016). Role of Plant Growth Promoting Rhizobacteria in Agricultural Sustainability-A Review. Molecules, 21 (5), 673-693.

Vicente, E. J. \& Dean, D. R. (2017). Keeping the nitrogen-fixation dream alive. Proceedings of the National Academy Sciences, 114 (12), $3009-3011$.

Zgadzaj, R., Garrido Oter, R., Jensen, D. B., Koprivova, A., Schulze Lefert, P. \& Radutoiu, S. (2016). Root nodule symbiosis in Lotus japonicus drives the establishment of distinctive rhizosphere, root, and nodule bacterial communities. Proceedings of the National Academy of Sciences, 113, 7996-8000. 